\title{
Obstructive Uropathy Due to an Incarcerated Ureteroinguinal Hernia
}

\author{
Erik Anderson ${ }^{\mathrm{a}, \mathrm{c}}$, Anthony Corcoran ${ }^{\mathrm{b}}$
}

\begin{abstract}
Ureteroinguinal hernia with or without ureteral incarceration resulting in obstructive uropathy is an especially uncommon case. Ureteroinguinal hernia should be included in the differential diagnosis when a hernia is detected on physical exam or found on imaging concurrent with new or unexplained hydronephrosis. The present case illustrates the importance of recognizing this condition. This patient, with a history of renal transplant, was asymptomatic other than a reducible inguinal hernia on exam and an elevated creatinine on lab work. Nephrostomy tube placement, an important temporizing measure to relieve obstruction, and subsequent ureteral reconstruction with inguinal hernia repair was successful in preserving the patient's transplant kidney function. The management described may be helpful in guiding future surgical approaches to similar scenarios.
\end{abstract}

Keywords: Ureteroinguinal hernia; Incarceration; Obstructive uropathy; Nephrostomy

\section{Introduction}

Obstructive uropathy caused by hernia-associated incarceration of the ureter is rare. Ureteral hernias were first described in 1880 and fewer than 140 cases have been described in the literature since that time [1], with even fewer describing associated incarceration and obstructive uropathy [2]. When present, the majority of cases are associated with inguinal hernias; however, incarceration is relatively uncommon due to the large

\footnotetext{
Manuscript accepted for publication June 23, 2015

aDepartment of Internal Medicine, Health Sciences Center T16-020, Stony Brook University Medical Center, 101 Nicolls Road, Stony Brook, New York 11794-8093, USA

bepartment of Urology, Health Sciences Center T9-040, Stony Brook University Medical Center, 101 Nicolls Road, Stony Brook, New York 11794 8093, USA

${ }^{c}$ Corresponding Author: Erik Anderson, 416 Midland Avenue, Yonkers, New York 10704-2759, USA. Email: eanderson22@nshs.edu
}

doi: http://dx.doi.org/10.14740/wjnu218w size of the majority of inguinal hernias [3]. Ureteroinguinal hernias have been reported in men and women; however, they are more common in men and typically occur in the fifth or sixth decade of life. They may be more common in patients with a history of kidney transplant given the location of the ureter within the space of Retzius. Inguinal ureteral hernias occur more frequently on the right side, likely due to differences in the morphology of the fascia of Toldt. On the left, the fascia of Toldt is at the level of the secondary root of the mesosigmoid, which may tighten the retroperitoneum and fix the ureter in some cases. This anatomical arrangement is not present on the right side $[4,5]$.

Two anatomical variations of ureteroinguinal hernia have been reported. The paraperitoneal type occurs in about $80 \%$ of cases, in which the ureter slides beside the peritoneal sac such that the ureter constitutes part of the hernia sac wall. This sac frequently contains other sliding organs, usually colon. The second and less common type is extraperitoneal, which is characterized by a lack of a peritoneal sac [6]. The herniated ureter is accompanied only by retroperitoneal fat [7]. Anomalies of the urinary system (for example, ptosis of the kidney) are common in these cases $(46 \%)$ and imaging of the urinary tract is indicated even when the ureteral hernia is incidentally discovered at surgery $[4,8]$. Both the paraperitoneal and extraperitoneal hernias are predominantly indirect.

Ureteroinguinal hernias may present clinically as abdominal pain and obstructive uropathy, but some patients are asymptomatic. Urologic symptoms may occur if there is coexisting bladder herniation, which is a rare phenomenon that also occurred in this case. Bladder involvement is rarely associated with ureteral hernia, likely because indirect hernia is more common than direct hernia ( $80 \%$ versus $20 \%$ ) in the setting of ureteral hernia. Herniation of the bladder usually occurs in direct hernias [5]. Symptoms that may be present in the case of bladder herniation are characteristic of bladder outlet obstruction, such as urinary frequency, obstructive symptoms or urinary retention, dysuria, nocturia, and hematuria.

The condition is often diagnosed incidentally during or after herniorrhaphy, when the ureter is inadvertently injured. This highlights the importance of preoperative diagnosis. Anterograde or retrograde urography, computed tomography (CT), or magnetic resonance imaging (MRI) can be used as diagnostic imaging studies. A ureteral hernia can be demonstrated on an anterograde nephrostogram by a redundant loop

Articles () The authors | Journal compilation (C) World J Nephrol Urol and Elmer Press Inc ${ }^{\mathrm{TM}}$ | www.wjnu.elmerpress.com

This is an open-access article distributed under the terms of the Creative Commons Attribution License, which permits unrestricted use, distribution, and reproduction in any medium, provided the original work is properly cited 


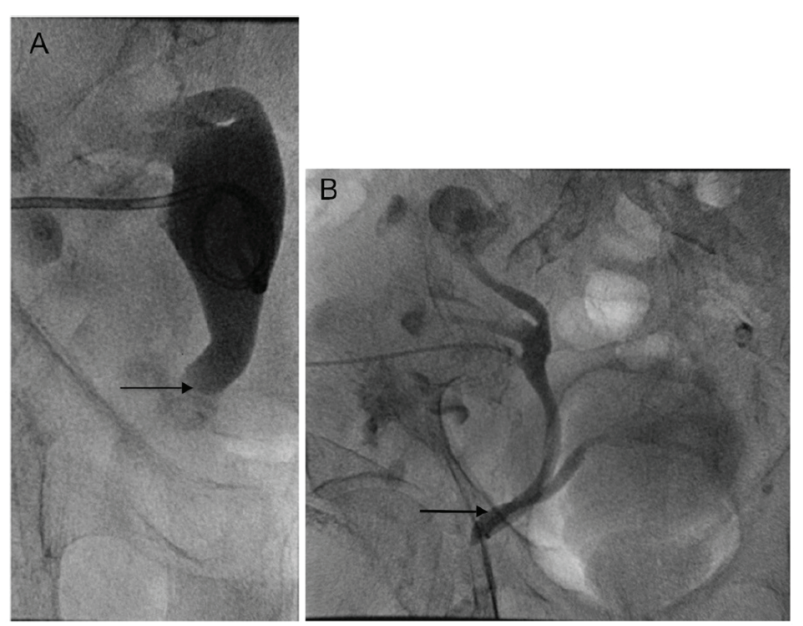

Figure 1. (A) Nephrostogram immediately before nephrostomy tube placement shows severe hydroureter to the level of the pubic ramus (arrow), proximal to the site of ureteral incarceration. (B) Nephrostogram performed after the nephrostomy tube placement 2 months prior shows the urinary tract to be patent, but barely. The arrow demonstrates the site of ureteral incarceration in the inguinal hernia. Tube nephrostogram showed good internal drainage of the transplant kidney, and extravasation of contrast into the bladder is demonstrated.

of ureter, called the "curlique" or "loop the loop" sign (Fig. $1 \mathrm{~B}$, arrow). This finding is pathognomonic of a ureteral hernia. The loop is oriented vertically in inguinal hernias, and horizontally in ureterosciatic hernias. CT or MRI with or without intravenous contrast can also make the diagnosis when the distal ureter is seen entering the inguinal canal or extending beyond the bony pelvis [6]. Furthermore, one must have a high index of suspicion for the diagnosis if there is new or unexplained hydronephrosis present ipsilateral to the hernia, or if hydronephrosis is present in the setting of a renal transplant. In these cases, CT urogram, diuretic renogram, or retrograde pyelogram may be indicated prior to surgical intervention.

Ureteroinguinal hernias are treated surgically because of the risk of obstructive uropathy. Herniorrhaphy with simple reduction of the ureter may be performed, or resection of the redundant ureter followed by primary anastomosis or ureteroneocystostomy $[9,10]$. In preparation for surgery, a nephrostomy tube should be placed for palliation of obstructive uropathy [6]. Intraoperatively, the herniated ureter must be carefully dissected away from the hernia sac and replaced in the retroperitoneal space to prevent ureteral injury. Postoperative ultrasound, CT urogram, or diuretic renogram should be done to ensure patency and proper replacement of the ureter $[9,10]$.

The present case conveys the importance of an accurate diagnosis of incarcerated ureteroinguinal hernia. Early recognition of the condition and appropriate treatment, illustrated by the following case description, is important in order to avoid renal failure.

\section{Case Report}

An 87-year-old male with a history of polycystic kidney dis- ease status-post bilateral nephrectomies and a living related donor transplant in 2001 presented with fevers and shortness of breath. He had left lower lobe crackles and a reducible right inguinal hernia. He was diagnosed with a lobar pneumonia and treated with intravenous antibiotics. On hospital day 3 , he developed an acute kidney injury (AKI), thought to be secondary to antibiotics versus dehydration, and was started on intravenous fluids. He subsequently developed respiratory distress secondary to pulmonary edema, and his renal function did not improve with fluids. An ultrasound to evaluate progressively worsening AKI revealed moderate to severe hydronephrosis of the right transplant kidney that was new compared to a prior CT scan of the abdomen/pelvis. The ultrasound also showed moderate hydroureter with non-visualization of the distal aspect of the ureter, suspicious for obstruction. A CT cystogram was performed to evaluate for obstruction and revealed marked hydronephrosis and hydroureter, demonstrating reflux into the collecting system as well as incarceration of a dilated ureter along with the anterior portion of the bladder within a fat-containing right inguinal hernia. Urology was consulted and the patient underwent a right nephrostomy tube placement. After relief of the obstruction, his renal function returned to baseline level. He was scheduled for ureteral reconstruction and right inguinal hernia repair.

At exploratory laparotomy, the right inguinal hernia was found to contain the right transplant ureter and a portion of the anterior bladder with the distal portion of the transplant ureter adherent to the inguinal hernia sac, indicative of a paraperitoneal hernia. Following ureterolysis of the right transplant ureter and preperitoneal repair of the right inguinal hernia, a right distal transplant ureterectomy and ureteroneocystostomy was performed over a ureteral stent with nephrostomy tube removal. At 2-month follow-up, the patient was asymptomatic with return of baseline renal function. No evidence of obstruction was noted with follow-up imaging done at 8 months.

\section{Discussion}

Obstructive uropathy following renal transplantation is not uncommon with an incidence of 3\% [7]. However, ureteral obstruction secondary to ureteroinguinal herniation with incarceration is a rare occurrence. The present case illustrates the importance of prompt recognition and proper surgical management. Failure in diagnosis and poor management can lead to loss of the allograft.

On review of the literature, there are only two cases of ureteroinguinal hernia occurring in a transplant graft [2, 7]. Only one of the two cases [7] involved incarceration of the ureter. The second patient [2] was asymptomatic on presentation with the physical exam significant only for a reducible hernia, similar to the present case. At initial workup the patient had graft hydronephrosis and an elevated creatinine, and subsequent magnetic resonance urography revealed an ureteroinguinal hernia.

The present case illustrates the importance of a high suspicion for ureteroinguinal hernia in patients with new or unexplained hydronephrosis located ipsilateral to the hernia or 
in the setting of a renal transplant. Incarceration of an ureteroinguinal hernia is a feared complication that results in obstructive uropathy and progressively worsening renal function without proper diagnosis and treatment. Importantly, ureteroinguinal hernia can be included in the differential diagnosis of obstructive uropathy when a hernia is detected on physical exam, as in the present case, or found on imaging.

This case also illustrates that nephrostomy tube placement is an important temporizing measure allowing relief of the obstruction without delay, while allowing subsequent surgical exploration under optimal conditions, also described by Osman et al in a similar case. Although stenting of the ureter at the time of definitive repair facilitates identification and protection during hernia repair, ureteral stent placement may be difficult given the length and tortuosity of ureter in these cases [11]. Furthermore, the resultant periureteral inflammation may make dissection of the ureter off surrounding structures more difficult than it already may be. In select cases, the hernia may be reduced under fluoroscopic control with the use of antegrade urography prior to hernia repair [7].

The case highlights successful management of an incarcerated ureteroinguinal hernia. Given the rarity of this condition, the management described will likely be helpful in guiding future approaches to the same scenario. The case should raise awareness of the condition for surgeons performing routine inguinal hernia repairs. Surgeons must be aware of the possibility of ureteroinguinal hernia in order to avoid ureteral injury during hernia repair, as preoperative recognition of the condition is paramount to a successful outcome.

\section{Disclaimer}

The views expressed in this article are the views of the author and not an official position of the institution.

\section{Disclosures}

No conflict of interest to declare; production of article was without financial support.

\section{References}

1. McKay JP, Organ M, Bagnell S, Gallant C, French C. Inguinoscrotal hernias involving urologic organs: A case series. Can Urol Assoc J. 2014;8(5-6):E429-432.

2. Verbeeck N, Niedercorn JB, Mc Intyre D, Pouthier D, Lamy S. Assessment of renal graft obstruction due to ureteral inguinal hernia: US detection and 3D MR confirmation. JBR-BTR. 2007;90(2):132-134.

3. Eilber KS, Freedland SJ, Rajfer J. Obstructive uropathy secondary to ureteroinguinal herniation. Rev Urol. 2001;3(4):207-208.

4. Sanchez AS, Tebar JC, Martin MS, Bachs JM, Moreno MJ, Navarro HP, Rodriguez JA. Obstructive uropathy secondary to ureteral herniation in a pediatric en bloc renal graft. Am J Transplant. 2005;5(8):2074-2077.

5. Giuly J, Francois GF, Giuly D, Leroux C, Nguyen-Cat RR. Intrascrotal hernia of the ureter and fatty hernia. Hernia. 2003;7(1):47-49.

6. He L, Herts BR, Wang W. Paraperitoneal ureteroinguinal hernia. J Urol. 2013;190(5):1903-1904.

7. Osman Y, Ali-El-Dein B, El-Leithy R, Shokeir A. Sliding hernia containing the ureter--a rare cause of graft hydroureteronephrosis: a case report. Transplant Proc. 2004;36(5):1402-1404.

8. Malde S, Bilagi P, Marsh H. Obstructive uropathy due to uretero-inguinal hernia: An uncommon occurrence. Indian J Urol. 2013;29(4):355-356.

9. Mantle M, Kingsnorth AN. An unusual cause of back pain in an achondroplastic man. Hernia. 2003;7(2):95-96.

10. Giglio M, Medica M, Germinale F, Raggio M, Campodonico F, Stubinski R, Carmignani G. Scrotal extraperitoneal hernia of the ureter: case report and literature review. Urol Int. 2001;66(3):166-168.

11. Won AC, Testa G. Chronic obstructive uropathy due to uretero-inguinal hernia: A case report. Int J Surg Case Rep. 2012;3(8):379-381. 